\title{
SOME APPLICATIONS OF LANDWEBER-NOVIKOV OPERATIONS ${ }^{1}$
}

\author{
DAVID M. SEGAL
}

\begin{abstract}
Previous results on the characteristic numbers of $S p$-manifolds are extended in three different ways. I. It is shown that the primitive symplectic Pontrjagin class evaluated on a $4\left(2^{j}-1\right)$ dimensional $S p$ manifold always gives a number divisible by 8 . This forms an analogue to a well-known result of Milnor concerning $U$-manifolds. II. It is shown that some of the results of Floyd as well as an analogue of the previous result can be obtained for 'pseudo-symplectic' manifolds. III. Results are generalised to $(S p, f r)$ manifolds.
\end{abstract}

1. $4\left(2^{j}-1\right)$ dimensional $S p$-manifolds. Let $s_{\pi}(p)[M], \pi$ a partition of $n=n(\pi), M$ a $4 n(\pi)$ dimensional stably symplectic manifold, denote the normal symplectic Pontrjagin number of $M$ corresponding to the $\pi$ symmetrised polynomial in a system of indeterminates for which the symplectic Pontrjagin classes are the elementary symmetric polynomials. Throughout this section we will set $k=2^{j}-1$ and $M$ will denote a $4 k$ dimensional stably symplectic manifold.

THEOREM 1.1.8|s $(k)(p)[M]$.

Remarks. 1. The unitary analogue, $2 \mid s_{(k)}(c)[N], N$ stably unitary is well known; it could be proven by the techniques used below.

2. The techniques of [3] are not adequate by themselves to prove Theorem 1.1 .

Proof. Actually we will prove slightly more: Let $\pi$ be any partition of $k$ all of whose parts are themselves integers of the form $2^{s}-1$. Then $8 \mid s_{\pi}(p)[M]$.

If $\pi=\left(a_{1}, \ldots, a_{r}\right)$, let $D(\pi)=\prod_{i}\left[\left(2 a_{i}+2\right) ! / 2\right]$. Well known fact. 2 । $\sum_{n(\pi)=k}\left(s_{\pi}(p)[M] / D(\pi)\right)$. This is the 'Todd genus' relation of Stong [4] who put things in an 'abnormal' form; using normal rather than tangential numbers makes computation manageable. In particular, we can see that for a fixed $k$ the denominators $D(\pi)$ with maximal number of factors of 2 will be just those for which all parts of $\pi$ are of the form $2^{s}-1$.

By Proposition 4 of [3] it is automatic that $4 \mid s_{\pi}(p)[M]$ for all $\pi, n(\pi)=k$. If we can show that $8 \mid s_{\pi}(p)[M]$ whenever $\pi=\left(a_{1}, \ldots, a_{r}\right), n(\pi)=k, r>1$

Presented to the Society, January 26, 1975; received by the editors November 1, 1974 and, in revised form, January 24, 1975.

AMS (MOS) subject classifications (1970). Primary 57A70; Secondary 55B20.

Key words and phrases. Landweber-Novikov operation, symplectic manifold, pseudo-symplectic manifold, symplectic-framed manifold.

1 This research was supported by the Research Foundation of the City University of New York.

(C) American Mathematical Society 1976 
and all $a_{i}$ of the form $2^{s}-1$, then it will follow from the above that 8 $s_{(k)}(p)[M]$.

Now assume inductively that Theorem 1.1 holds in dimensions less than $4 k$; by [4] it certainly holds in dimensions 4 and 12 . Let $\bar{\pi}=\left(a_{1}, \ldots, a_{r}\right)$ be a partition of $k$ with $r>1$ and all $a_{i}$ of the form $2^{s}-1$. Since $\bar{\pi}$ is a partition of an odd number into odd parts there is a number $k^{\prime}$ which occurs exactly $f$ times as a part of $\bar{\pi}, f$ odd. Let $\bar{\pi}^{\prime}$ denote the partition obtained from $\bar{\pi}$ by deleting one occurence of $k^{\prime}$. Let $S\left(\bar{\pi}^{\prime}\right)$ denote the symplectic LandweberNovikov operation corresponding to $\bar{\pi}^{\prime}$. Then from the results of [1] on the action of such operations,

$$
s_{\left(k^{\prime}\right)}(p)\left[S\left(\bar{\pi}^{\prime}\right) M\right]=f s_{\bar{\pi}}(p)[M]+\sum_{\pi} a\left(\pi, \bar{\pi}, \bar{\pi}^{\prime}\right) s_{\pi}(p)[M],
$$

where the summation on the right runs through all partitions $\pi$ obtained by adding $k^{\prime}$ to one of the parts of $\bar{\pi}^{\prime}$, and the coefficients $a\left(\pi, \bar{\pi}, \bar{\pi}^{\prime}\right)$ are integers which are in fact even as a consequence of the fact that the parts of $\bar{\pi}^{\prime}$ are all of the form $2^{s}-1$. Then by Proposition 4 of [3] (and since $n(\pi)$ is odd), 8 divides the summation term. But by the inductive hypothesis, 8 divides the left side of (1.2). Our assertion and the theorem then follow from the oddness of $f$.

2. Pseudo-symplectic manifolds. We call a $U$-manifold pseudo-symplectic if some nonzero multiple of its class in $M U_{*}$ is in the image of $M S p_{*}$; this will be the case if and only if every Chern number of the manifold involving an odd Chern class vanishes. Let $P s_{*}$ be the subring of $M U_{*}$ consisting of such classes. Let $j, p, d$ be the maps in the cofibration sequence of spectra

$$
M S p \stackrel{j}{\rightarrow} M U \stackrel{p}{\rightarrow} M U / M S p \stackrel{d}{\rightarrow} S M S p .
$$

There is a well-defined map $h_{*}^{P s}: P s_{*} \rightarrow H_{*}(M S p)$ obtained by restricting the Hurewicz homomorphism $h_{*}^{M U}: M U_{*} \rightarrow H_{*}(M U)$ to $P s_{*}$ and then composing with $j_{*}^{-1}$. We regard the sympletic Pontrjagin numbers as defined on $P s_{*}$. Note that $\operatorname{Im} h_{*}^{S p} \subset \operatorname{Im} h_{*}^{P_{s}} \subset H_{*}(M S p)$ (inclusions strict) and that $\operatorname{Im} h_{*}^{P s} / \operatorname{Im} h_{*}^{S p}$ gives the torsion elements of $\operatorname{Im} p_{*}$ in the $(M U, M S p)$ long exact bordism sequence.

LemMA 2.1. Let $S(\pi): M S p \rightarrow S^{4 n(\pi)} M S p$ be a symplectic LandweberNovikov operation. Then we can find some U-bordism operation $T: M U$ $\rightarrow S^{4 n(\pi)} M U$ such that $T \circ j=S^{4 n(\pi)} j \circ S(\pi)$.

Proof. Treat $S^{4 n(\pi)} j \circ S(\pi)$ as a class in $M U^{4 n(\pi)}(M S p)$. Now $d_{*}\left(S^{4 n(\pi)} j \circ S(\pi)\right)=0$ in $M U^{4 n(\pi)+1}(M U / M S p)$ (since that group is trivial), so by exactness there must exist $T \in M U^{4 n(\pi)}(M U)$ such that $j_{*}(T)$ $=S^{4 n(\pi)} j \circ S(\pi)$.

This 'compatibility' lemma implies that $\operatorname{Im} h_{*}^{P s}$ is closed under the action of the symplectic Landweber-Novikov operations.

THEOREM 2.2. Let $M$ be a $4 k$ dimensional pseudo-symplectic manifold. Then

(i) $2 \mid s_{\pi}(p)[M]$ if $n(\pi)$ is odd or if $\pi=\left(2^{j}\right)$;

(ii) $4 \mid s_{(k)}(p)[M]$ if $k=2^{j}-1$.

REMARK. Floyd first studied pseudo-symplectics (they are the 'related 
manifolds' of the title of [2]) and part (i) was proved by him by rather different methods.

Proof. Exactly as for symplectics in [3] except that one has weaker low-dimensional divisibility properties to feed into the machinery so that statements involving 4 become statements involving 2 while those involving 2 become vacuous. By the same token, part (ii) is done on the model of Theorem 1.1 above.

3. ( $S p, f r)$ manifolds. Let $h_{*}^{S p, f r}: M S p / f r_{*} \rightarrow H_{*}(M S p / f r)$ be the Hurewicz map for $M S p / f r$, the spectrum representing $(S p, f r)$ bordism. We wish to obtain divisibility conditions on characteristic numbers of $(S p, f r)$ manifolds. One would expect to use a compatibility lemma which showed that $\operatorname{Im} h_{*}^{S p, f r}$ is closed under the action of symplectic Landweber-Novikov operations, find some 'starting' conditions and proceed as with the symplectic and pseudosymplectic cases.

Actually something happens which makes our work easier (and our results stronger). If $n(\pi)>0$ then $S(\pi)$ can be lowered to a map $S(\pi)^{\prime}: M S p / f r$ $\rightarrow S^{4 n(\pi)} M S p$ so that $S(\pi)$ actually sends the $(S p, f r)$ classes into full-fledged $S p$-classes. Thus all the divisibility conditions of [3] hold equally for $(S p, f r)$ manifolds except in the starting dimensions:

THEOREM 3.1. Let $M$ be a $4 k(S p, f r)$ manifold. Then

(i) $4 \mid s_{\pi}(p)[M]$ if $n(\pi)>1$ and odd or if $\pi=\left(2^{j}\right), j>1$;

(ii) $2 \mid s_{\pi}(p)[M]$ if $n(\pi)>2$ and $\equiv 2$ (4) or if $\pi=\left(2^{j}, 2^{j}\right), j>1$.

The proof of Theorem 1.1 does not carry over to the $(S p, f r)$ case.

\section{BIBLIOGRAPHY}

1. J. F. Adams, S. P. Novikov's work on operations on complex cobordism, University of Chicago Lecture Notes, 1967.

2. E. E. Floyd, Stiefel-Whitney numbers of quaternionic and related manifolds, Trans. Amer. Math. Soc. 155(1971), 77-94. MR42 \#8509.

3. D. M. Segal, Divisibility conditions on characteristic numbers of stably symplectic manifolds, Proc. Amer. Math. Soc. 27(1971), 411-415. MR42 \#5282.

4. R. E. Stong, Some remarks on symplectic cobordism, Ann. of Math. (2)86(1967), 425-433. MR36 \#2162.

Department of Mathematics, City College, City University of New York, New York, NEW YORK 10031

Current address: Department of Mathematics, New York Institute of Technology, Old Westbury, New York 11568 